\title{
DISEÑO Y CONSTRUCCIÓN DE UNA BOBINA TESLA DE 1680 W, PARA LA ENSENAANZA DE CONCEPTOS BẤSICOS EN SISTEMAS ELÉCTRICOS DE POTENCIA
}

\section{DESIGN AND CONSTRUCTION OF A TESLA COIL $1680 \mathrm{~W}$, AS A TOOL FOR TEACHING BASIC CONCEPTS IN ELECTRIC POWER SYSTEMS}

\section{Juan Carlos Castro Galeano ${ }^{1}$ María Luisa Pinto Salamanca² Maira Fernanda Amaya Quitián 3}

\section{Resumen}

La Bobina de Tesla es un tipo de transformador resonante, funciona a elevadas frecuencias y eleva la tensión, construida con dos bobinas acopladas eléctricamente en un núcleo de aire la cual produce efectos observables por el ojo humano como chispas y descargas eléctricas. Este artículo presenta el diseño y construcción de una Bobina de Tesla de $1680 \mathrm{~W}$ con una distancia de ruptura de $1 \mathrm{~m}$ de longitud, la cual puede ser empleada como herramienta didáctica y pedagógica para la enseñanza de los conceptos fundamentales de sistemas eléctricos de potencia, como: efecto corona, resonancia de circuitos RLC, campos eléctricos y magnéticos, transmisión de energía sin conductores y alta tensión.

Palabras clave: aislamiento eléctrico, efecto corona, descargas eléctricas, transformador deTesla.

\section{Abstract} Recibido: agosto 29 de 2014
Aceptado: octubre 24 de 2014

Tesla coil is a type of resonant transformer, which operates at high frequencies and raises the voltage; it's built with two coils electrically coupled into an air core which produces observable effects by the human eye as sparks and electrical discharges. This article presents the design and construction of a 1680 W Tesla coil with a rupture distance of one meter length. The coil could be used as a teaching learning tool in the teaching of the fundamental concepts of electrical power systems as: corona effect, resonance RLC circuits, electric and magnetic fields, energy transmission without conductors and high voltage.

Key words: electrical insulation, corona effect, electrical discharges, Tesla transformer.

\footnotetext{
1 Ingeniero Electricista, Magíster en Ingeniería. Docente Escuela de Ingeniería Electromecánica, Universidad Pedagógica y Tecnológica de Colombia, Facultad Seccional Duitama.

2 Ingeniera Electrónica, Magíster en Ingeniería. Docente Escuela de Ingeniería Electromecánica, Universidad Pedagógica y Tecnológica de Colombia, Facultad Seccional Duitama.

3 Ingeniera Electromecánica. Escuela de Ingeniería Electromecánica, Universidad Pedagógica y Tecnológica de Colombia, Facultad Seccional Duitama, E-mail:mafe78@gmail.com

1,2,3 Grupo de Investigación y Desarrollo en Sistemas Electromecánicos GridsE, E-mail:gridse.uptc@gmail.com
} 


\section{Introducción}

Nikola Tesla, un extraordinario ingeniero SerbioAmericano, descubrió el principio del campo magnético rotatorio en 1882 e inventó los motores de inducción y el sistema trifásico de generación y distribución de electricidad, fundamentando las bases de la energía eléctrica y los sistemas eléctricos de potencia. Gracias a esto, grandes cantidades de energía eléctrica pueden ser generadas y transmitidas a grandes distancias, desde las plantas generadoras hasta ciudades y centros de consumo.

Uno de sus inventos más memorables es la Bobina de Tesla, la cual es un transformador con núcleo de aire, formado por dos devanados, un primario y un secundario, los cuales son circuitos resonantes compuestos cada uno por una inductancia y una capacitancia que se encuentran a una resonancia específica (Rajvanshi, 2007, p.5). Con este dispositivo proyectaba transmitir la energía eléctrica sin necesidad de conductores, y aunque en esta época su invento no prosperó, los sistemas de comunicación inalámbrica y telefonía celular actuales se basan en este principio, actualmente el IEEE considera a Tesla uno de los doce apóstoles de la ciencia eléctrica y anualmente entrega un reconocimiento en su nombre en el campo de la potencia eléctrica (Vujic, 2001, p. 324).

Este dispositivo resulta ser muy útil a la hora de la enseñanza de diferentes conceptos del área eléctrica, como: efecto corona, frecuencia de resonancia en circuitos RLC, alta tensión, conducción e ionización del aire, transformadores elevadores, entre otros, y se convierte en una herramienta didáctica que a su vez es muy atractiva por sus impactantes destellos luminosos. Igualmente se puede emplear para realizar pruebas al aislamiento de equipos eléctricos (Phung, 1991; Pungsiri y Chotig, 2008; Sels et al., 2002).

Este artículo presenta el diseño, construcción y puesta a punto de una Bobina de Tesla de $1680 \mathrm{~W}$, con una distancia de ruptura de un metro de longitud; la bobina puede ser empleada como herramienta didáctica y pedagógica para la enseñanza de los conceptos fundamentales de sistemas eléctricos de potencia. A continuación se detallan el cálculo de los diversos componentes de la bobina de Tesla, junto con el proceso de fabricación y pruebas realizadas. Como resultado se obtuvo un arco de $40 \mathrm{~cm}$ y en la prueba con un tubo fluorescente, desde un metro de distancia, se iniciaron destellos luminosos en su interior.

\section{Materiales y métodos}

Para el diseño y construcción de los elementos de la bobina se empleó la metodología sugerida por, (Tilbury, 2008, p. 11-235), haciendo más comprensible su funcionamiento. A continuación se describen los diversos componentes de la bobina deTesla y su circuito equivalente.

\subsection{Circuito equivalente de la bobina de Tesla}

El circuito típico de la bobina de Tesla se presenta en la figura 1, en la cual:

T1: Transformador de alimentación elevador 120/15000V

R1: Resistencia limitadora

$\mathrm{Cp}$ :Condensador primario

Cp:Condensador secundario

Lp:Devanado primario

Ls: Devanado secundario

Spark Gap: Interruptor en aire

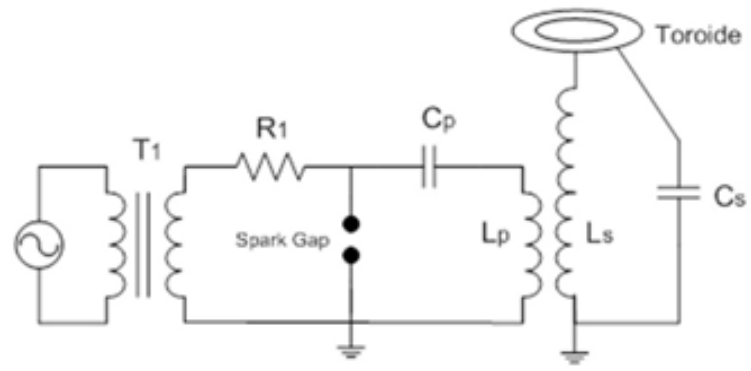

Figura 1. Circuito básico de la bobina de Tesla

El principio de funcionamiento de la bobina de Tesla se basa en un circuito resonante, donde la 
energía del transformador elevador T1 es empleada para cargar el condensador de alto voltaje $\mathrm{Cp}$. El alto voltaje al cual se ven sometidos los electrodos del spark gap, hace que se produzca en el aire a su alrededor una ionización generando un efecto de avalancha, que hace que el aire entre los gap's sea un conductor. Así, la energía almacenada en el condensador se descarga en el devanado primario Lp, en forma de oscilación de alta frecuencia, la cual es exactamente la frecuencia de resonancia entre los devanados primario y secundario de la bobina, con lo que se genera un alto voltaje en el terminal de descarga del devanado secundario, toroide, formando arcos de descarga hacia el electrodo de tierra. Este proceso de transferencia de energía de un circuito a otro continúa de forma cíclica, mientras se presenten rupturas en el sparkgap.

\subsection{Transformador de alimentación (T1)}

Se diseñó y construyó un transformador tipo seco con las siguientes características:

IAT: $130 \mathrm{~mA}$

VAT: $15000 \mathrm{~V}$

IBT: 16,67 A

VBT:120V

S: $2 \mathrm{kVA}$

P: $1950 \mathrm{~W}$

El devanado de alta tensión está formado por 26386 espiras, de alambre magneto calibre 31 AWG, distribuidas en ocho galletas y a su vez dividida en dos devanados para ser conectados en paralelo. El devanado de baja tensión posee 106 espiras en calibre 12 AWG en doble conductor, distribuidas en dos capas. El aislamiento del devanado de alta tensión se reforzó con resina Royal-POX y fibras Nómex de 0,25 mm entre galletas, ver figura 2 .

Considerando que el transformador va a estar sometido a esfuerzos eléctricos, éste se opera a una tensión de $12000 \mathrm{~V}$ para aumentar su vida útil. Para la selección del tipo de núcleo, construcción de los devanados y distancias de aislamiento y refrigeración se tomó la metodología del (MIT, E.E Staff, 2003, p. 151-285)

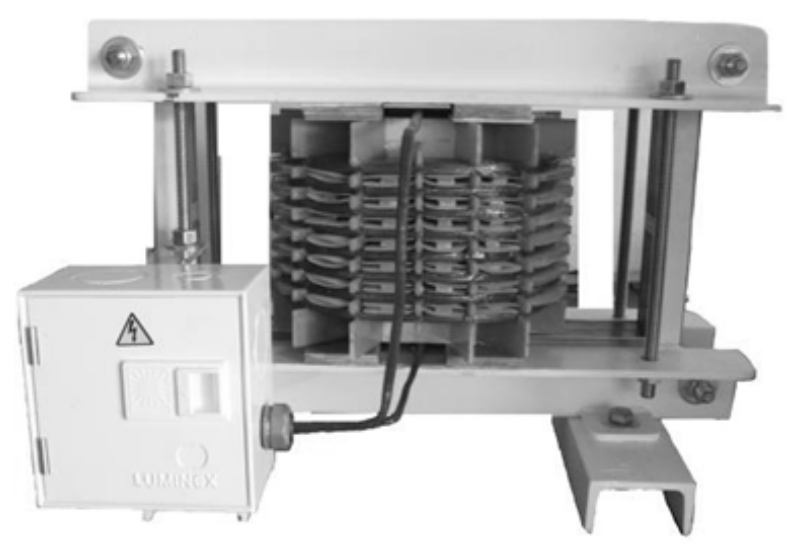

Figura 2. Transformador de alimentación T1

\subsection{Condensador Primario (Cp)}

Para la frecuencia de $60 \mathrm{~Hz}$, la tensión de $12000 \mathrm{Vy}$ la corriente de 0,13 A en el lado de AT del transformador $\mathrm{T} 1$, se calculó el valor de la capacitancia del condensador primario $\mathrm{Cp}$ empleando la ecuación (1)

$$
C p=\frac{I}{2 \pi \times f_{\text {linea }} \times V}
$$

Donde:

flinea: Frecuencia de línea, $\mathrm{Hz}$

V:Tensión, $\mathrm{V}$

I:Corriente, $\mathrm{A}$

$C p$ :Capacitancia primaria, $\mathrm{F}$

$$
C p=\frac{0,13}{2 \pi \times 60 \times 12000}=0,0287 \mu F
$$

El condensador se fabricó envolviendo dos flejes de aluminio de $0,2 \mathrm{~m} \times 2 \mathrm{~m}$ de longitud sobre un tubo de PVC, separados por un aislamiento compuesto por una fibra Tríplex en medio de dos fibras Nómex y dos fibras diamantadas, como se representa en la figura 3. 


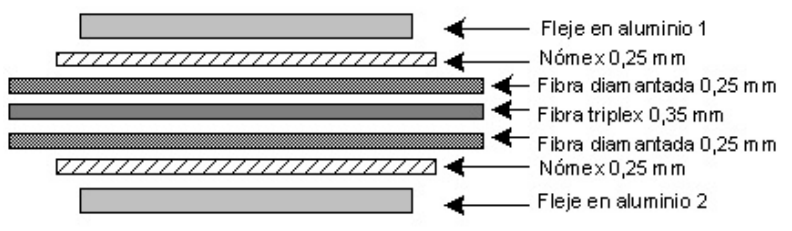

Figura 3. Esquema del aislamiento del condensador

El voltaje de ruptura estimado para el aislamiento empleado, se considera de $34 \mathrm{kV}$. Los voltajes de ruptura en $A C$ de los aislamientos empleados son: 2500V , 5000V y 19000 V para las fibras Nómex, Diamantada y Triplex respectivamente.

El proceso de fabricación del condensador se ilustra en la figura 4.
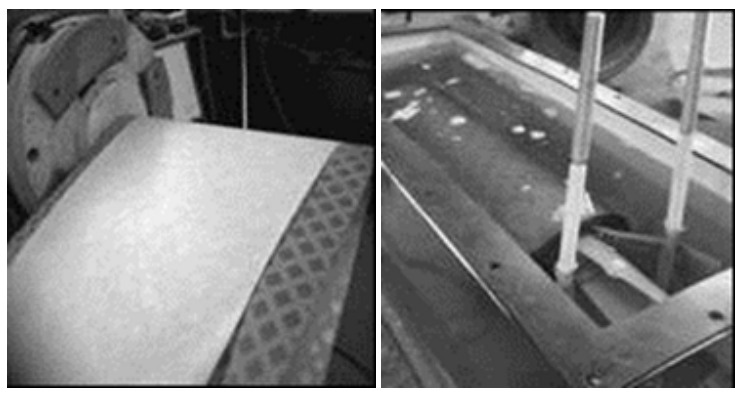

Figura 4. Fabricación del condensador $C p$

En la parte inicial de cada fleje se realizaron las salidas para ser conectadas a los bujes, realizados en teflón mediante maquinado en torno y en su interior con bulones de bronce. Una vez construido el condensador se sumergió en aceite dieléctrico para mejorar el aislamiento del mismo, verfigura 5 .

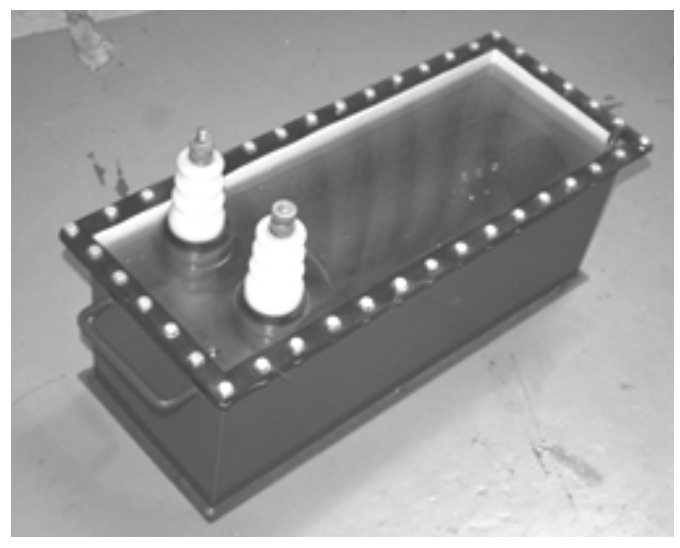

Figura 5. Condensador de alta tensión sumergido en aceite dieléctrico
Una vez construido $C P$, su valor medido empleando un multímetro FLUKE 187, fue de $0,017 \mu \mathrm{F}$, menor al valor calculado; por lo anterior fue necesario recalcular los demás componentes de la bobina.

\subsection{Bobina secundaria, Ls}

El secundario de la bobina de Tesla (alta tensión) se realizó en alambre magneto calibre 18 AWG con un total de 1100 espiras, enrolladas sobre un tubo de PVC de 16,81 cm de diámetro externo. Se posicionó de forma vertical y en la parte superior se ubicó el toroide en aluminio, para formar el terminal de alta tensión y a su vez distribuir uniformemente la capacitancia a tierra. El cálculo de la altura se realizó empleando la ecuación (2):

$$
H=N \times d
$$

Donde:

H: Altura de la bobina, $m$

$N$ : Número de espiras, 1100

$d$ : Diámetro del conductor de la bobina, $m$

El diámetro del alambre 18 AWG fue de 1,024 mm, portanto:

$$
H=1100 \times 0,001024=1,13 m
$$

Para el cálculo de la inductancia Ls se empleó la ecuación (3):

$$
L s=\frac{0,156 A^{2} N^{2}}{3,54 A+3,94 H} \times 0,001
$$

Donde:

A: Radio de la bobina, $\mathrm{cm}$

$N$ : Número de espiras de la bobina.

$\mathrm{H}$ : Altura de la bobina, $\mathrm{cm}$

$$
L s=\frac{0,16 \times 8,45^{2} \times 1100^{2}}{3,54 \times 8,45+3,94 \times 113} \times 0,001=29 \mathrm{mH}
$$


La capacitancia de la bobina en pF, se calculó empleando la ecuación (4), donde Hy A en metros

$$
\begin{aligned}
& C s=7,48 H+16,14 A+76,38 \sqrt{\frac{A^{3}}{H}} \quad(4) \\
& C s=7,48 \times 1,13+16,14 \times 0,0845+76,38 \sqrt{\frac{0,0845^{3}}{1,33}}=12 p F
\end{aligned}
$$

\subsection{Toroide}

Este terminal se ubica en la parte superior de la bobina secundaria de Tesla, comportándose como una capacitancia que completa el circuito de alta tensión, debido a que la autocapacitancia propia de la bobina no es suficiente y necesita ser reforzada. Cuando el circuito empieza a funcionar, la tensión es tan alta que llega un punto en que el terminal superior no puede contener mas carga y se produce la ruptura, la cual se manifiesta en un arco eléctrico.

En el diseño de esta bobina se seleccionó un toroide con diámetro exterior $\mathrm{D} 1=0,508 \mathrm{~m}$ y un diámetro del toroide $\mathrm{D} 2=0,1016 \mathrm{~m}$; para calcular su efecto hacia tierra, se empleó la ecuación (5).

$C_{t}=2,8 \times\left(1,2781-\frac{D_{1}}{D_{2}}\right) \times \sqrt{\frac{2 \pi^{2} \times\left(D_{1}-D_{2}\right) \times \frac{D_{2}}{2}}{4 \pi}}$

Para la elaboración del toroide se analizaron diferentes materiales, pero debido a que es recomendable emplear una superficie lisa, se optó por realizarlo en aluminio, para lo cual fue necesario construir un molde en madera para su fundición y su posterior mecanizado conservando sus dimensiones, figura 6. Una vez fabricado el toroide, éste fue ubicado sobre la bobina y se midió su capacitancia hacia tierra con un equipo DOBLE M4000, presentando un valor de 33,41 pF.

\subsection{Bobina primaria Lp}

Para el diseño de la bobina primaria, de baja tensión, se calculó la frecuencia de resonancia. Cuando los circuitos de alta y baja tensión

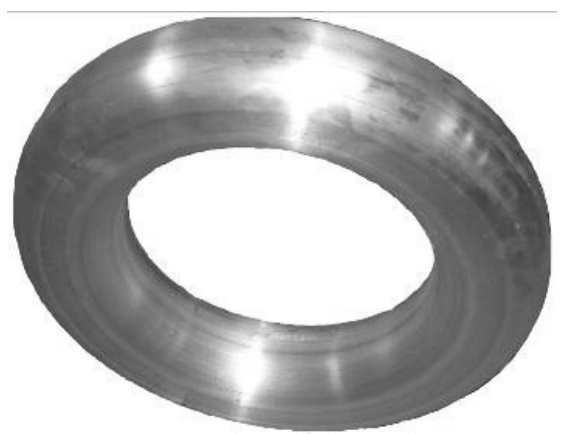

Figura 6. Toroide en aluminio fundido

alcanzan la misma frecuencia, se eleva la tensión en la bobina secundaria, produciendo la ionización del aire y los arcos eléctricos, desde el toroide a los elementos conectados a tierra, con una distancia menor a $1 \mathrm{~m}$ desde el mismo.

Empleando la ecuación (6) se calculó la frecuencia de la bobina secundaria fos, donde $L s$, en $[H]$ y Ctotalen [F].

$$
f_{o s}=\frac{1}{2 \pi \times \sqrt{L_{s} \times C_{\text {total }}}}
$$

Donde Ctotal considera la capacitancia del toroide de la bobina secundaria y el transformador T1, valor medido empleando equipo M4000:

$$
f_{\text {os }}=\frac{1}{2 \pi \times \sqrt{29 \times 10^{-3} \times 111,53 \times 10^{-12}}}=89,8 \mathrm{kHz}
$$

Para el cálculo de la resonancia de la bobina de Tesla, se igualaron la frecuencia de la bobina secundaria fos y primaria fop, y a partir de esta igualdad se calculó la inductancia de la bobina primaria Lp empleando la ecuación (7), con $f_{o p}=$ $f_{o s}$ :

$$
\begin{gathered}
\frac{1}{2 \pi \times \sqrt{L_{s} \times C_{\text {total }}}}=\frac{1}{2 \pi \times \sqrt{L_{p} \times C_{p}}} \\
L_{p}=\frac{L s \times C_{\text {total }}}{C_{p}}(7) \\
L_{p}=\frac{29 \times 10^{-3} \times 111,53 \times 10^{-12}}{17 \times 10^{-9}}=185 \mu \mathrm{H}
\end{gathered}
$$


Con este valor se calculó de forma iterativa el número de espiras de la bobina primaria empleando las ecuaciones (8) y (9):

$$
L p=\frac{N^{2} \times R}{30 R-11 D i},[\mu \mathrm{H}]
$$

Donde

N: Número de espiras del devanado primario

$R$ :Radio externo de la bobina primaria, $\mathrm{cm}$

Di:Diámetro interno de la bobina primaria, $\mathrm{cm}$

$$
R=\frac{D i+N(w+S)}{5,08},[\mathrm{~cm}]
$$

Donde:

w: Radio medio geométrico, $\mathrm{cm}$

\section{S:Espacio entre espiras}

Asumiendo un espaciado entre espiras de 1,52 cm, un conductor rectangular de radio medio geomérico de $1,18 \mathrm{~cm}$ y un diámetro interno de 23 $\mathrm{cm}$ se encontró la configuración para la bobina primaria.

Número de vueltas: 18

Diámetro interno: $22,86 \mathrm{~cm}$.

Diámetro externo: $105,3 \mathrm{~cm}$.

Espacio entre espiras: $1,5 \mathrm{~cm}$.

Para la elaboración de la bobina primaria, se fabricaron separadores en baquelita en forma de peinilla, ubicando en cada ranura una espira. Se empleó un conductor rectangular de 2,9 x 11,45 $\mathrm{mm}$, de cobre aislado con cinta Kapton. La bobina fabricada se presenta en la figura 7.

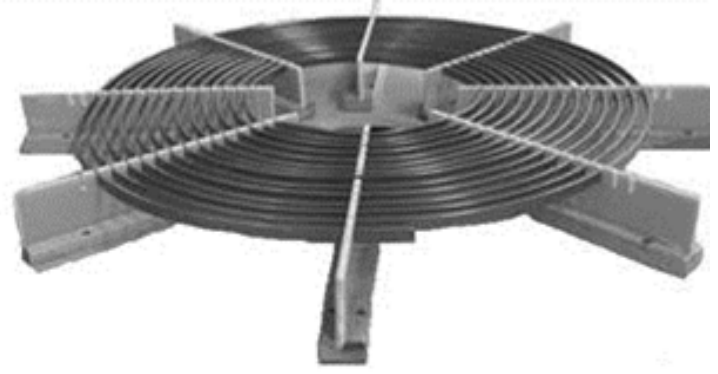

Figura 7. Bobina de baja tensión terminada

\subsection{Spark Gap}

El spark gap es el interruptor de alta potencia, encargado de iniciar la descarga en el circuito de alta tensión de T1 (primario de la bobina de Tesla). Está compuesto por unos electrodos separados una pequeña distancia en aire, el cual se ioniza cuando la tensión supera un umbral. Para su construcción se evaluaron varias alternativas, seleccionando uno del tipo dinámico y rotatorio, el cual depende de la velocidad de un motor para cambiar la frecuencia de resonancia de la bobina deTesla.

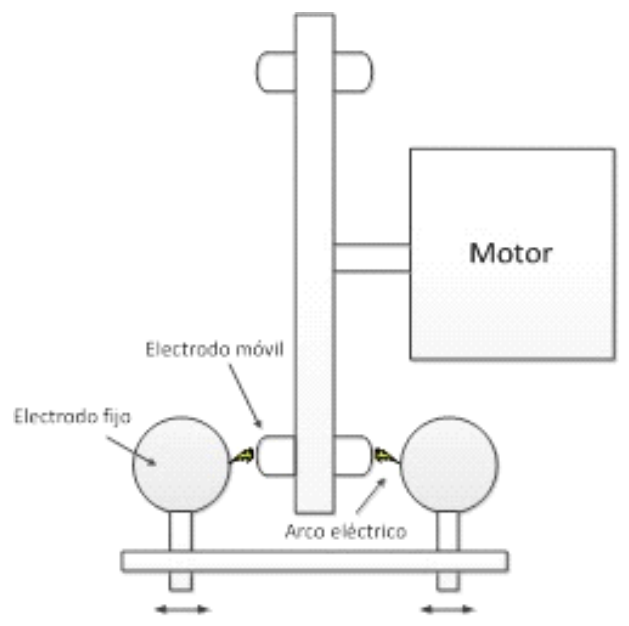

Figura 8. Interruptor en aire (spark gap)

En el eje del motor se acopló un disco aislante en baquelita sobre el cual se ubicaron cuatro electrodos iguales, y en la base se instalaron dos electrodos fijos a igual distancia de los electrodos móviles para que la ruptura fuera simultánea en ambos lados. Todos los electrodos se fabricaron en aluminio con terminación redondeada para controlar mejor la descarga simulando una ruptura entre electrodos planos. Los electrodos fijos se realizaron con un sistema de ajuste horizontal con el fin de variar la distancia y permitir el cambio de la tensión de ruptura, figura 8.

El motor fue soportado sobre una estructura metálica como presenta la figura 9, asegurando la 
altura adecuada para evitar saltos eléctricos desde los terminales energizados del spark gap hacia la estructura de la mesa.

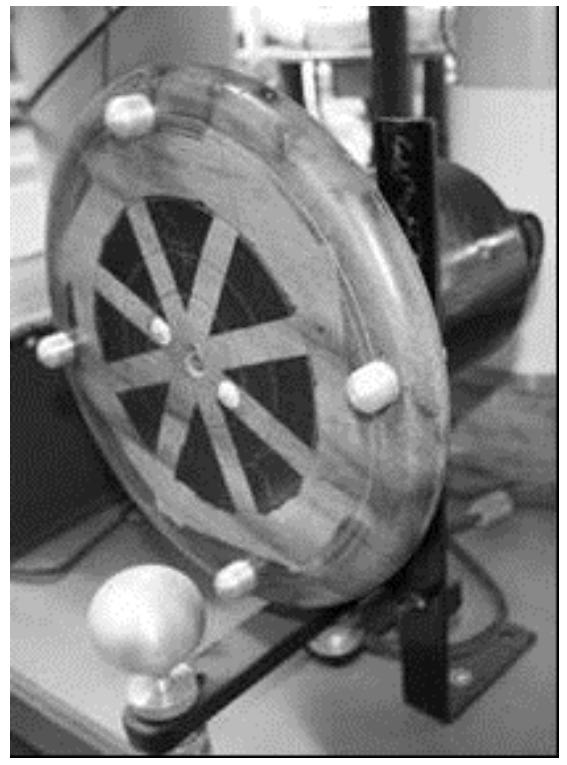

Figura 9. Spark gap construido

\subsection{Resistencia limitadora $\mathrm{R} 1$}

Para evitar corrientes elevadas en el transformador T1 en los instantes de operación del spark gap, se instaló una resistencia limitadora a la salida de su terminal de alta tensión. Esta resistencia se calculó simulando un corto en el lado de alta tensión de T1. Considerando que la corriente en este devanado es de $130 \mathrm{~mA}$, su impedancia de cortocircuito de $9,24 \%, 6,82 \mathrm{k} \Omega$ en las bases de $12 \mathrm{kV}$ y $2 \mathrm{kVA}$ y empleando el circuito de la figura 10 , se obtuvo una resistencia limitadora de 85,5 $\mathrm{k} \Omega$.

La resistencia limitadora se fabricó en agua empleando un tubo de PVC, como se muestra en la figura 11.

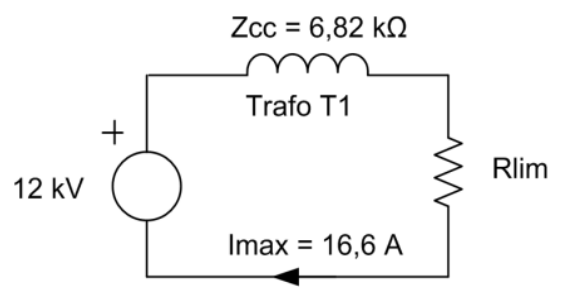

Figura 10. Cálculo de la resistencia limitadora R1

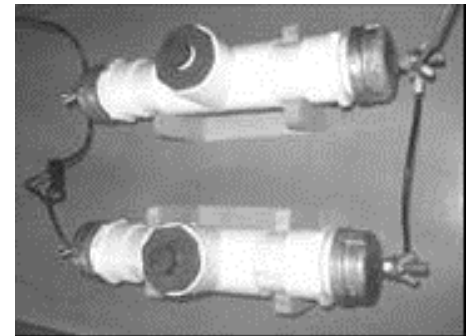

Figura 11. Resistencia limitadora

\section{Resultados y discusión}

Previo al ensamble final de la bobina, se verificó el correcto funcionamiento de sus componentes.

\subsection{Pruebas del transformadorT1}

Se realizó la prueba de resistencia óhmica de los devanados, resistencia de aislamiento y relación de transformación presentando resultados satisfactorios. Se midió la impedancia de corto circuito energizando el transformador por el lado de AT con en devanado de BT en corto, los valores medidos se presentan en la tabla 1. La impedancia de corto circuito se calculó empleando la ecuación (8):

$$
\begin{aligned}
& Z_{C C}=\frac{V_{\mathrm{Pr}}}{V_{n}} \times \frac{I_{n}}{I_{\mathrm{Pr}}} \times 100 \\
& Z_{C C}=\frac{435}{15000} \times \frac{130 \times 10^{-3}}{0,0408} \times 100=9,24 \%
\end{aligned}
$$

\begin{tabular}{cccc}
\hline \multicolumn{2}{c}{$\begin{array}{c}\text { Valores nominales lado } \\
\text { de AT }\end{array}$} & \multicolumn{2}{c}{$\begin{array}{c}\text { Valores medidos por } \\
\text { el lado de AT }\end{array}$} \\
\hline V, [V] & I, [mA] & V, [V] & I, [A] \\
15000 & 130 & 435 & 0,0408 \\
\hline
\end{tabular}

Tabla 1. Datos para el cálculo de la impedancia de corto circuito

Finalmente se realizó la prueba de factor de potencia y capacitancia del aislamiento como se sugiere en (Castro, 2009, p. 4), con el objetivo de medir las capacitancias del transformador T1 para realizar los ajustes en la frecuencia de resonancia en el circuito de la bobina de Tesla. La capacitancia AT contra BT y tierra, vista desde el circuito de la bobina deTesla, fue de 101,23 pF. 


\subsection{Ensamble final de la bobina de Tesla}

Se construyó una mesa de 1,2m x 1,2m, con los bordes metálicos y las estructuras horizontales en madera, para soportar todos los componentes de la bobina de Tesla. Las conexiones entre los elementos se realizaron lo más cortas posibles y se emplearon terminales para evitar desconexiones accidentales. El ensamble final se presenta en la figura 12.

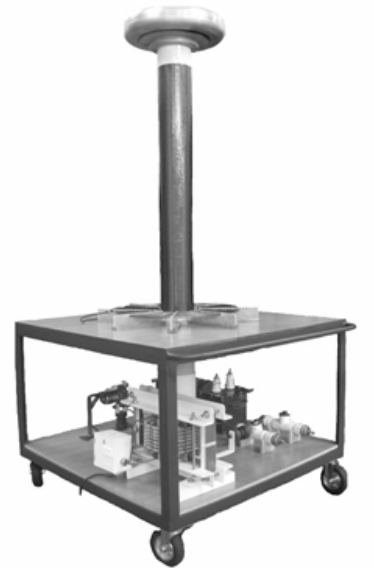

Figura 12. Bobina de Tesla ensamblada

\subsection{Pruebas finales}

Una vez ensamblada, se energizó la bobina y se obtuvo un arco de $40 \mathrm{~cm}$, como se muestra en la figura 13. La distancia de ruptura fue menor a la esperada, lo cual se debe en gran parte a las condiciones de temperatura, humedad y presión atmosférica, condiciones que disminuyen la capacidad de los equipos eléctricos de alta tensión.

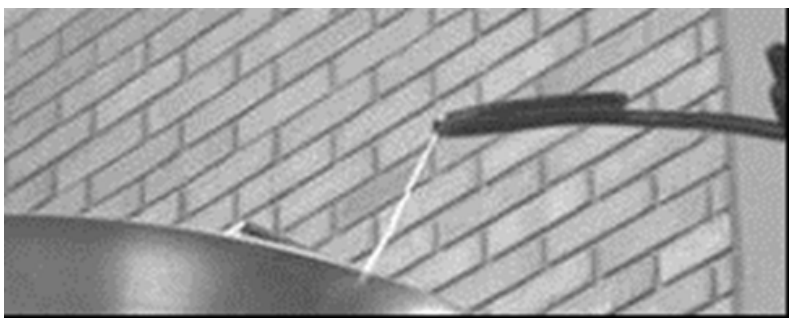

Figura 13. Arco generado

Se realizó la prueba con un tubo fluorescente y desde $1 \mathrm{~m}$ de distancia se iniciaron destellos de luz en su interior, los cuales aumentan en intensidad a medida que se acerca al toroide.

Dado el riesgo que existe al trabajar con sistemas de alta tensión, para realizar las conexiones del circuito de la bobina, es necesario que el equipo se encuentre desenergizado y el condensador sea descargado; la manipulación de alto voltaje y cargas eléctricas almacenadas pueden ser letales. Durante el funcionamiento de la bobina se producen campos eléctricos y magnéticos considerables, que pueden afectar el funcionamiento de equipos electrónicos sensibles; por ello se recomienda a las personas con marcapasos observar los destellos luminosos, producidos por la bobina, a una distancia considerable.

\section{Conclusiones}

Aunque el diseño de la Bobina de Tesla fue una actividad sencilla, su construcción fue compleja ya que debido a que sus componentes no se consiguen comercialmente, fue necesaria la construcción de cada uno de ellos. Una vez finalizada la construcción de todos sus elementos, se encontró que los valores medidos en algunos de ellos se alejaron de los esperados, desplazando de esta forma la frecuencia de resonancia de la bobina y disminuyendo los efectos luminosos, siendo necesario encontrar de forma experimental los ajustes y modificaciones para una nueva frecuencia realizando cambios en el número de espiras de la bobina de baja tensión, variando la velocidad del spark gap y cambiando los valores de la resistencia limitadora, la cual regula el tiempo de carga del condensador.

La bobina de Tesla aquí presentada permite un mejor análisis y comprensión de los conceptos fundamentales de los sistemas eléctricos de alta tensión, como: el efecto corona, resonancia en circuitos RLC, campos eléctricos y magnéticos y la transmisión de energía eléctrica sin conductores. Lo anterior debido a que estos conceptos se pueden explicar de forma práctica por medio de la 
Bobina de Tesla como herramienta didáctica de apoyo para la enseñanza de los fenómenos eléctricos: Además, podrá ser empleada para realizar pruebas en equipos aisladores y transformadores cuando son sometidos a sobretensiones transitorias.

Adicionalmente, el laboratorio de Electricidad de la Universidad Pedagógica y Tecnológica de Colombia, sede Duitama, cuenta con una bobina de Tesla para mejorar la enseñanza y la investigación del área eléctrica con la cual se beneficiarán todos los estudiantes de las áreas de electricidad de la Universidad ya que ninguna de las sedes cuenta con una bobina de este tipo.

\section{Agradecimientos}

Los autores agradecen a la Dirección de Investigaciones de la Universidad Pedagógica y Tecnológica de Colombia, por la financiación del proyecto: Diseño y construcción de una bobina tesla, para el laboratorio de electricidad de la UPTC sede Duitama; código SGI-873. Igualmente agradecen el apoyo técnico para la construcción de la bobina, a la empresa Industrias Explorer en Duitama, Boyacá.

\section{Referencias}

Castro, J. C. (2009). Pruebas de diagnóstico en transformadores. Congreso Internacional en Alta Tensión y aislamiento Eléctrico ALTAE 2009, Medellín noviembre de 2009.
M.I.T, E.E. STAFF (2003). Staff de Ingenieria Electrica (Electrical Engineering Staff) del Massachusetts Institute of Technology MIT. "Circuitos magnéticos y transformadores". España: Editorial Reverté.

Pungsiri, B., Chotig, S. (2008). The Compact Tesla Transformer for Testing Pin Insulator. International Conference on Condition Monitoring and Diagnosis, Beijing, China, April 2124.

Rajvanshi, A. K. (2007). Nikola Tesla - The creator of the Elecric Age. Resonance Journal of Science education, India: Bangalore.

Sels, T., Karas, J., Lopez-Roldan, J., Declercq, D., Dommelen, V., \& Belmans, R. (2002). Electrical Insulation Behaviour Subject to Fast Transients using a Tesla Transformer. Power and Energy Systems (PES 2002), Marina del Rey, USA, May 1315, 2002.

Tilbury, M. (2008). The Ultimate Tesla Coil Design and Construction Guide. United States: McGraw Hill.

Vujic, J. (2001). Nikola Tesla: 145 of Visionary Ideas. Congreso TELSLIKS 2001, Septiembre de 2001. (VUJIC, 2001, p. 324). 


\section{INSTRUCCIONES PARA LOS AUTORES (Revista de Investigación, Desarrollo e Innovación) ISSN: 2027-8306}

\section{PRESENTACIÓN}

La Revista de Investigación, Desarrollo e Innovación es el medio de divulgación científica de la Facultad Seccional Duitama, y tiene como objetivo la publicación de las investigaciones, reflexiones y adelantos generados en diversas áreas del saber, tanto de nuestra institución como de otras con las cuales se mantiene lazos de colaboración. Dado el carácter multidisciplinar de la Facultad, la Revista IDI publica trabajos en las siguientes temáticas: Diseño e Ingeniería, Educación y Administración.

La Revista IDI publica artículos originales de investigación, reflexión y revisión, preferentemente en las áreas prioritarias de la revista y con interés para la comunidad académica regional, nacional e internacional, escritos en idiomas español einglés.

La revista tiene una periodicidad semestral y se editan dos números por año. La recepción de los manuscritos es permanente y puede hacerse en cualquier momento.

El Comité Editorial establece dos fechas de corte para determinar los artículos aceptados para la publicación: 15 de Abril para el primer semestre y 15 de octubre en el segundo semestre.

De acuerdo con los criterios de indexación dados por COLCIENCIAS, los artículos de investigación, reflexión y revisión se definen de la siguiente manera:

Artículo de investigación científica y tecnológica. Documento que presenta, de manera detallada, los resultados originales de proyectos terminados de investigación. La estructura generalmente utilizada contiene cuatro apartes importantes: introducción, metodología, resultados y conclusiones.
Artículo de reflexión. Documento que presenta resultados de investigación terminada desde una perspectiva analítica, interpretativa o crítica del autor, sobre un tema específico, recurriendo a fuentes originales.

Artículo de revisión. Documento resultado de una investigación terminada, donde se analizan, sistematizan e integran los resultados de investigaciones publicadas o no publicadas, sobre un campo del conocimiento, con el fin de dar cuenta de los avances y tendencias de su desarrollo. Se caracteriza por presentar una cuidadosa revisión bibliográfica de por lo menos 50 referencias.

\section{INSTRUCCIONES PARA LA PREPARACIÓN DE MANUSCRITOS}

Los artículos postulados a la revista deben tener una extensión máxima de 20 páginas.

La digitación debe realizarse en fuente de letra Times New Roman de 12 puntos, a doble espacio, una columna y todas las márgenes de $2 \mathrm{~cm}$ en hoja tamaño carta. Evite el uso de viñetas, notas al pie y pies de página, ya que estos elementos no son aceptados para la diagramación.

El título del artículo deberá ser corto o dividido en título y subtítulo, atractivo para el lector potencial y escrito en mayúscula sostenida, no más de 15 palabras. Igualmente se debe incluir la traducción del título al idioma ingles.

Luego se incluyen el nombre completo de los autores acompañado con la filiación: título de pregrado y postgrado, cargo e institución donde labora, grupo de investigación, dirección y correo electrónico.

Posteriormente se incluirá un resumen en español de máximo 200 palabras con su respectiva 
traducción al inglés, cuatro palabras claves en español y cuatro en inglés.

Para la presentación del contenido se recomienda la utilización de varios subtítulos enumerados, iniciando con uno de introducción y finalizando con otro de conclusiones. Según la naturaleza del trabajo sería deseable contar con las siguientes secciones: 1. Introducción, 2. Materiales y métodos, 3. Resultados y discusión, 4. Conclusiones, agradecimientos si los hay y Referencias

Cuando el manuscrito sea resultado de un proyecto de investigación o de un proceso formativo a nivel de pre- o postgrado, deberá explicitarse esta situación en el apartado de Agradecimientos, indicando el código del proyecto y la entidad financiadora, o el nombre del programa de formación en el cual se inscribe el proyecto de grado, respectivamente.

Todas las figuras y tablas deberán realizarse en escala de grises, ser referenciadas dentro del texto, numerarse y titularse de manera clara. Además, deben localizarse en el lugar más cercano a donde son citadas por primera vez. Cuando se trate de figuras, deberá garantizarse una buena resolución para su reproducción en cualquier tipo de papel, mínimo 150 dpi.

Todas las figuras, a saber: imágenes, esquemas, fotografías, etc, deberán incluirse como imágenes y no como autoformas de Word.

Las tablas deben ser editadas desde Word y no insertadas como imagen.

Cuando los artículos incluyen ecuaciones, estas deben ser elaboradas en un editor de ecuaciones apropiado, por ejemplo el editor de ecuaciones de Microsoft.

Todas las referencias listadas en la sección de bibliografía deben ser citadas a lo largo del texto, de la siguiente forma:

Para un autor: (apellido, año)

Para dos autores: (apellido primer autor y apellido segundo autor, año)

Para más de dos autores: (apellido, et al., año)

Para cita de cita: [fuente original] (apellido, año), citado por [fuente a la que se tiene acceso] (apellido, año). Otra forma es: de acuerdo con [fuente original] apellido (año), citado por [fuente a la que se tiene acceso], (apellido, año).

Ejemplo: "La interacción social según Goffman (1972), citado por (Rizo García, 2006), afirma que (.........".

La interacción social es la realización rutinaria de los encuentros (Goffman, 1972), citado por (Rizo García, 2006)".

Las referencias bibliográficas serán situadas al final del texto en orden alfabético, en la sección de bibliografía así:

\section{Libro con dos autores}

Pérez, R. y Gallego, R. (1996). Corrientes constructivistas. Bogotá: Editorial magisterio.

\section{Artículo en revista}

Müller, T., Pfennig, A., Klein, P., Gradl, G., Jäger, M. \& Schnelle. T. (2003). The potential of dielectrophoresis for single-cell experiments. IEEE in Medicine and Biology Magazine, 22, 51 -61.

Ponencias o conferencias en simposio, congreso, reuniones, etc.

Sandoval J., Salamanca W., Cardozo V., Duarte J. E. y Fernández Morales F. H. (2006). Desarrollo de un inversor monofásico didáctico. VII Congreso de Tecnologías Aplicadas a la Enseñanza de la Electrónica TAEE'06. (pp. 72 - 77). Madrid, España: Universidad Complutense de Madrid. 
(2004). Modelo de Evaluación de Software Educativo. Extraído el 13 de mayo de 2008, de http://investigacion.ilce.edu.mx/dice/proyectos/ evaluacion/modelo.htm.

Es responsabilidad de los autores el brindar la información completa de las referencias bibliográficas utilizadas y dar los créditos correspondientes. Aunque se trate de citas de trabajos propios deberá darse el crédito respectivo.

Todo artículo será evaluado de forma anónima por dos pares evaluadores, designados por el Comité Editorial. Las excepciones a este requisito serán decididas por tal comité.

Los documentos deben ser entregados al siguientecorreo electrónico:

revista.idi@gmail.com.

Los documentos irán acompañados de una carta remisoria donde se especifique el autor de contacto.

El contenido de las contribuciones es entera responsabilidad de los autores, y de ninguna manera de la revista o de las entidades para las cuales trabajan los autores. Se entiende que el material enviado a la Revista IDI no ha sido publicado ni enviado a otros órganos de difusión cualquiera sea su tipo.
El Comité Editorial se reserva el derecho de modificar el título de los artículos y realizar los cambios editoriales que considere pertinentes, para dar al artículo la mayor claridad posible. Por lo tanto, se recomienda a los autores escribir con el mayor rigor, verificando la ortografía, empleando párrafos cortos y homogéneos, y utilizando, adecuadamente, los signos de puntuación.

Los autores ceden a la Revista la propiedad de sus derechos de autor, para que los artículos sean publicados y comunicados en cualquier forma o medio. Los documentos se enviarán a bases de datos y sistemas de indización, para su divulgación y se podrán consultar a través de la Internet, en la página web de la Revista.

Con el envío de manuscrito a la revista, los autores aceptan las condiciones establecidas en las presentes normas para autores.

\section{CONTACTO}

Universidad Pedagógica y Tecnológica de Colombia

Facultad Seccional Duitama

Centro de Investigación y Formación Avanzada CIFAD

Carrera 18 Calle 23

Duitama, Boyacá Colombia

Tel:098 7604100 ext. 2823

E-mail:revista.idi@gmail.com

revista.idi@uptc.edu.co

www.uptc.edu.co/enlaces/rinvduitama 